2019-11-07

\title{
"I feel like only half a man"
}

\section{Patel, D}

http://hdl.handle.net/10026.1/16035

$10.1145 / 3359184$

Proceedings of the ACM on Human-Computer Interaction

Association for Computing Machinery (ACM)

All content in PEARL is protected by copyright law. Author manuscripts are made available in accordance with publisher policies. Please cite only the published version using the details provided on the item record or document. In the absence of an open licence (e.g. Creative Commons), permissions for further reuse of content should be sought from the publisher or author. 


\title{
"I feel like only half a man": Online Forums as a Resource for Finding a "New Normal" for Men Experiencing Fertility Issues
}

\author{
DILISHA PATEL, University College London, UK \\ ANN BLANDFORD, University College London, UK \\ MARK WARNER, University College London, UK \\ JILL SHAWE, University of Plymouth, UK \\ JUDITH STEPHENSON, University College London, UK
}

\begin{abstract}
Infertility can place a significant burden on couples and individuals when trying to conceive. Approximately $20-30 \%$ of all cases of infertility are due to male-related factors. Whatever the cause of difficulty in conceiving, little is known about how men find support when dealing with fertility issues, or when or how online resources are being used. This paper reports on a qualitative study of anonymous online posts $(N=603)$ from forums related to fertility that are used by men. We analysed this data using thematic analysis to understand how men are using online forums as a resource when experiencing fertility issues. We found that online forums play a valued role in facilitating connections between men experiencing an often stigmatised condition. These forums offer men accessible and private spaces which allow for more open discussion, helping them to make sense of their situation. We discuss our findings in relation to Genuis and Bronstein's model of finding a "new normal" and present our elaborated model of finding a "new normal" in the context of experiencing fertility problems.
\end{abstract}

CCS Concepts: • Human-centered computing $\rightarrow$ Empirical studies in HCI; HCI theory, concepts and models.

Additional Key Words and Phrases: Online Forums; Online Communication; Men; Fertility; Masculinity; Stigma; Finding a New Normal; Sense-making

ACM Reference Format:

Dilisha Patel, Ann Blandford, Mark Warner, Jill Shawe, and Judith Stephenson. 2019. "I feel like only half a man": Online Forums as a Resource for Finding a "New Normal" for Men Experiencing Fertility Issues. Proc. ACM Hum.-Comput. Interact. 3, CSCW, Article 1 (November 2019), 20 pages. https://doi.org/00.0000/0000000.0000000

\section{INTRODUCTION}

There is a growing interest in understanding the role $\mathrm{HCI}$ can play in supporting people experiencing issues around fertility. Whilst infertility affects both men and women [7, 36], approximately $20-30 \%$ of fertility issues arise from male-related factors [1]. Existing work on digital tools for fertility and trying to conceive, such as fertility tracking apps [23], has predominantly focused on women.

Authors' addresses: Dilisha Patel, University College London, 66-72 Gower Street, London, WC1E 6EA, UK, dilisha.patel@ ucl.ac.uk; Ann Blandford, University College London, 66-72 Gower Street, London, WC1E 6EA, UK, a.blandford@ucl.ac.uk; Mark Warner, University College London, 66-72 Gower Street, London, WC1E 6EA, UK, mark.warner@ucl.ac.uk; Jill Shawe, University of Plymouth, Drake Circus, Plymouth, PL4 8AA, UK, jill.shawe@plymouth.ac.uk; Judith Stephenson, University College London, 74 Huntley Street, London, WC1E 6AU, UK, judith.stephenson@ucl.ac.uk.

Permission to make digital or hard copies of all or part of this work for personal or classroom use is granted without fee provided that copies are not made or distributed for profit or commercial advantage and that copies bear this notice and the full citation on the first page. Copyrights for components of this work owned by others than ACM must be honored Abstracting with credit is permitted. To copy otherwise, or republish, to post on servers or to redistribute to lists, requires prior specific permission and/or a fee. Request permissions from permissions@acm.org.

(c) 2019 Association for Computing Machinery.

2573-0142/2019/11-ART1 \$15.00

https://doi.org/00.0000/0000000.0000000

Proc. ACM Hum.-Comput. Interact., Vol. 3, No. CSCW, Article 1. Publication date: November 2019. 
Less attention is paid to supporting men, or to men's experiences of infertility. This may be because issues around fertility are often difficult to discuss due to the internalised stigma that it can create, especially around male-factor infertility [26]; this stigma can compromise well-being and mental health [21]. This, together with the paucity of research in this area, limits our ability to support men who face fertility difficulties.

Previous research suggests that men often choose to access health related information from social and collaborative sources online, due to ease of access and concerns relating to privacy [39, 64]. Other research has shown that men are likely to use websites and online forums as a source of information and support relating to their personal health [9, 20, 22]. Although expressions of personal feelings and emotional needs on digital platforms are strongly influenced by gender norms [18], there is little research that focuses specifically on how men use online platforms to discuss their health and health related needs. This can be due to difficulties in identifying where men are engaging in these sensitive conversations, as they can be hidden due to fear of judgement and threats to men's feelings of masculinity [28].

Through the inclusion of men into the body of HCI research on fertility, we aim to give men a voice in issues around fertility, especially around male-factor infertility. Without this more gender balanced understanding, the lack of research on the role of men in these processes could exacerbate the unequal focus on women as primary caregivers [2], and maintain societal norms of women bearing the burden of responsibility around fertility.

This study builds on previous work on how men engage with online digital resources to share experiences with other men [2,3]. Our work extends into the field of fertility and, in doing so, we develop insights into this under-supported group.

The focus of this research was to better understand how men use online forums when experiencing fertility difficulties. We find online spaces provide an environment which enables men to share with one another in order to elicit personalised advice and emotional support which aids the process of sense-making during an often emotionally disruptive time. From our analysis of online digital forums, this paper presents a number of contributions to the $\mathrm{HCI} / \mathrm{CSCW}$ community.

Our work provides insight into how men are using online forums to communicate with one another when undergoing fertility treatment, a socially stigmatised and often hidden experience. We apply the finding a "new normal" model [27] to the context of infertility, which provides us with a better understanding of the different stages of information seeking of men dealing with conception difficulties. We provide a new perspective on how men use online forums as a resource for sense-making to find their "new normal" through interactions with others in anonymous online communities. In doing so, we present an elaborated model of finding a "new normal" to the context of infertility.

\section{RELATED WORK}

This study is informed by previous work on men's experiences of (in)fertility, primarily from the health and psychology domains, and on online communication about stigmatising topics from the $\mathrm{HCI}$ and CSCW communities.

\subsection{Men and Fertility}

Our work builds on existing work from the CSCW community that has explored how men use social media to share their experiences with one another around issues related to fatherhood $[3,4]$. Findings reported by Ammari and Schoenebeck [3,4] highlight the need to include a focus on men in research on reproductive health and child rearing. We focus on the early stages of the fertility journey that couples may experience together: difficulties when trying to conceive. 
There is substantial evidence outside the CSCW community establishing the importance of men preparing for conception by addressing their preconception health (e.g., [25, 37, 59]), and recent research has focused on men dealing with infertility (e.g., [7, 33]. Infertility, defined as the inability to conceive after 12 months of unprotected intercourse, affects up to $15 \%$ of couples worldwide; male factor infertility is solely responsible in almost $30 \%$ of all cases of infertility, but contributes to approximately $50 \%$ of infertility cases globally [1], affecting almost 10 million couples.

Studies have shown that men who are experiencing fertility difficulties may be willing to share and be expressive in environments that are deemed safe and confidential [33]. Malik and Coulson [43] report that such men openly discuss a range of feelings and concerns. They also show how forums can promote the exchange of support for those who experience infertility [44]. However, whilst this research along with others [34] have explored how users share their feelings online, little research has focused on men's specific needs and their information journey when they experience fertility difficulties.

There is a need to explore how and why this population seek support online to improve their health and well-being, in order to better understand their information seeking behaviours and requirements for support when they struggle to conceive; this is the focus of this paper.

\subsection{Online Health Forums}

Online forums are web-based discussion boards; these can consist of several topics and conversations, and forums are usually labelled to identify the topics of conversation that are within scope [60]. This increases their transparency and ability to attract users with similar interests. Within these forums registered users can write a statement, question, or share anything they choose about the specified topic in the form of a post. Other users can then reply to these posts forming a conversation thread. Each thread will have a title to identify the topic of conversation. Most forums have a moderator to ensure posts are abiding by the forum's rules and are sharing appropriate content.

Online forums are often used to create a community of like-minded individuals. These can be for specific health conditions, (e.g., managing HIV [14]), for advice (e.g., on physical activity [63]), or sharing experiences (e.g., of pregnancy [30]). Members exchange information with the expectation of having an empathetic anonymous audience [44]. Forums are also used to obtain low-cost, health related information or advice [67], and for disseminating information [60].

Men who are trying to conceive and experience infertility may lack contact with others in the same position due to location, the prevalence of the condition and disclosure norms [66]. Because online forums have dedicated spaces for groups of peers, they can "provoke personal understanding" and provide coping tools and safe spaces for discussion and sharing [10]. Previous studies have shown that some men use online forums to discuss personal, private and potentially sensitive topics [34, 38].

Online forums also give people an alternative way in which to communicate with their peers that may be preferable to face-to-face interactions [52]. Some men can find it difficult to speak openly or share thoughts or concerns with their peers and family due to the assumed stigma that surrounds infertility and the perceived masculinity associated with fertility and virility [34]. Posting in anonymous online forum groups can provide a safe and supportive environment for men who are preparing for conception or encountering difficulties with their fertility [18, 44].

\subsection{Stigmatising Conversations}

Gannon et al. [26] articulate the interplay between masculinity and infertility and how this can lead to feelings of stigmatisation. They describe the relationship as between "the denial of weakness or vulnerability, emotional and physical control, the appearance of being strong and robust, dismissal 
of any need for help" and virility. It is reported that since fatherhood is proof of masculinity, anything that threatens that can bring feelings of shame [24]. Hegemonic masculinity is used to describe the dominant characteristics men are often expected to exhibit in society [21], which can be affected when men experience infertility. This can hinder health information seeking behaviours as well as limiting men's avenues for emotional support as they report feeling stigmatised by their infertility [26, 32].

When a condition is stigmatised it can be difficult to openly share or reach out for support $[15,61]$. This can drive sensitive conversations into more anonymous and private spaces [42]. This in itself can indirectly contribute towards the stigma associated with the topics discussed through the lack of open and public conversation. It can be difficult to break these societal and cultural norms that enable the conversations to remain hidden, and lead to the creation of marginalised populations [8].

Stigma was once defined as "being a mark on someone of questionable moral status" [28]. Its definition has developed to include personal characteristics such as sexual orientation, health status, income or even nationality. Male-factor infertility can be a cause of internalised stigma [26], especially as trying to conceive is not often openly disclosed. It is commonly connected to negative responses such as the fear of judgement or prejudice [28]. Those that experience this form of stigmatisation are more likely to turn to online methods for information, support from peers and sharing to mitigate the potential consequences of openly identifying themselves to their personal networks $[8,10]$.

Anonymous online forums may be a preferred space for men who are trying to conceive and encounter infertility to share their experiences and feelings. It has been shown that providing a safe space for marginalised populations is important as it can enable the sharing of personal and sensitive information whilst protecting a person's privacy and, as a result, their well-being [41].

Therefore, we use online forums as a source of data on stigmatising conditions. This source of data has been previously used to better understand different aspects of health around stigmatised populations (e.g., $[14,16])$.

\subsection{Finding a "New Normal"}

When encountering an unexpected life event or health concern, there is often a need to make sense of the life disrupting event or new reality [47]. This can lead to the need for a return to normalcy: to their previous selves before the life disruption [55]. Alternatively, it can result in the need to understand what is normal for them now: defined as their "new normal". This premise has been reported in multiple domains and health conditions in parallel, making its origins unclear - for example, in cancer [35, 55], chronic conditions [13], bereavement [47] and mental health conditions [27].

The phenomenon of trying to establish a "new normal" has been widely discussed in the context of cancer survivorship. This varies from managing cancer treatment [6], dealing with the aftermath of physically altering surgery [35], as well as dealing with the rupture of self-image and wanting to feel normal within themselves and in the eyes of others after treatment [19].

Burgess et al. [13] discuss the information work that patients with chronic conditions undertake. The concept of finding a "new normal" emerges when patients discover a gap in their understanding or current knowledge. The authors present the concept of meaning-making as a crucial part of the patient journey in order to enable patients to prospectively plan what they want their future normal to be and act accordingly in the present. The act of meaning-making is key in this journey as this helps to infer what the life disruption means to them personally and to take this meaning into the formation of their future "new normal".

Massimi et al. [47] also depict the need for a "new normal" to be a consequence of a break down of routine. They report that the occurrence of new circumstances and perhaps new social surroundings

Proc. ACM Hum.-Comput. Interact., Vol. 3, No. CSCW, Article 1. Publication date: November 2019. 
can result in the acceptance of the concept. They explored this phenomenon using three case studies of bereavement, domestic violence and homelessness. Each of these characteristically different experiences produces a substantial life disruption and illustrates how technology and new social communities are used to facilitate the creation of a "new normal".

The experience of coming to terms with an unexpected diagnosis is described by Genuis and Bronstein [27], who note that normality is most commonly used to describe a situation in which there is an absence of disease or ill-health. Making sense of a health condition that disrupts routine is important, especially if it has come on suddenly or unexpectedly, which can lead to the requirement to make sense of a "new normal" post diagnosis. These feelings are clearly mirrored in the infertility population, as it is often described as an unexpected situation. However, it is not known how the fertility journey, which can be dynamic as well as lonely and isolating [32, 36], can be mapped on the information journey presented by these authors.

The literature describes a process in which people often look for similar others for advice and for comparison to explore what is acceptable in their new circumstance, rather than what is typical, when trying to understand their "new normal" following a life disruption [49]. This supports Genuis and Bronstein's postulation that it is important to find information and validate thoughts and knowledge with similar others. In conditions such as infertility, which are not typically openly discussed, it can be difficult to identify a community of others who are undergoing a similar journey. Therefore, online forums are a clear space where these communities have the opportunity to congregate, share and compare themselves with others.

We explore how men communicate in online forums and how this may influence their information journey to form their "new normal" when experiencing fertility problems.

\section{METHODOLOGY}

Conducting research with stigmatised populations can be problematic as participants are often difficult to reach and challenging to recruit [41]. As more people seek online resources for support around health, data disclosed online can provide unique insights into these populations. People may also be more open to sharing their feelings online as they often do not need to reveal their true identity before interacting [8]. Using online data is a less intrusive method of gathering first-hand accounts on users' experiences, feelings and needs [18]. As such, we chose to analyse online forum data to achieve our proposed research contributions, carefully considering the ethical implications in the design of this study (see: section 3.3).

\subsection{Data Collection}

Online searches were conducted using a search engine in an "incognito window", which is a web-browser setting that prevents internet browsing history from being stored. This was done to limit the influence previous searches or stored data would have on the results returned. The following search terms were used to locate online forums where men may be discussing preparing for conception and experiencing fertility difficulties: "forums for men ttc" (trying to conceive), "forums for fathers/dads discussing pregnancy planning", "forums for preparing for conception/ pregnancy", "forums for fertility", "forums for men/prospective fathers", "forums men planning pregnancy", "forums for low sperm count and/or poor sperm quality" and "forums for men/fertility". Whilst there are a number of conditions that can contribute to male factor infertility, it is most commonly defined using parameters of sperm health [21], this was the only causal search term used.

Forums that were dedicated to (in)fertility and trying to conceive and were publicly available to be viewed without registration were explored. These included forums that had dedicated spaces for men to communicate with one another. 
The forums chosen each had moderators who were responsible for ensuring the forum rules were adhered to. Users could register and sign-up using pseudonyms, enabling them to preserve their anonymity if they so wished. There were also options for users to send direct messages to one another, making private conversations possible.

An online web scraping application was developed using Scrapy [57], the open source web crawling framework in Python. This was used to extract comments from the identified online forums. We scraped the first 10 pages from each forum identified to ensure we had a sample of the most recent insights. We reached data saturation through the analysis of this sub-sample of posts.

3.1.1 Inclusion and Exclusion Criteria. Threads that were initiated by men and discussed topics or provided information relating to men's health, needs and requirements when preparing for conception, fatherhood or managing fertility were included in this analysis. In this manuscript we use the term "men" to refer to all people who self-identify as men and discuss trying to conceive to become fathers in the forum comments.

Threads that did not focus on the topic of fertility were excluded. If the thread was clearly initiated by a woman, it was excluded from the analysis, as it could not be reliably inferred to represent men's needs.

\subsection{Data Analysis}

The extracted threads were exported and anonymised where necessary. The first post in each thread was read and initially categorised into one of the following categories: "Written by men", "Written by women" [excluded], "Off Topic" [excluded], or "Further Review", for threads which remained unclear from the first post. In these cases, the thread was read in its entirety to determine whether to include it in the analysis. Threads which were "Off Topic" typically did not discuss preparing for conception or fertility difficulties, or were advertisements for research or products.

In most cases, it was evident whether a man or woman had authored the post based on its content, as users would often self-identify with their sex and age then talk about their reproductive conditions openly. E.g.: "I'm a 37-year-old man..."; "My husband and I have been TTC for 3 years."; "The first semen analysis I took...". It was equally clear when women were writing on behalf of or asking for advice for their male partner.

The included threads were then formatted and imported into NVivo 12. A thematic analysis was conducted [12], focusing on exploring how men use online forums when preparing for conception and experiencing fertility difficulties. We explored men's informational needs when posting on online forums.

All of the eligible data was read by the first author to aid familiarisation. This was followed by coding of the first post in each thread to establish the apparent need of the original poster. This first round of coding classified the posts into broad themes. Manual coding was then used to analyse threads for further themes and to aid in further immersion. The manually developed codes were descriptive and detailed. Examples of these were, "User has unexplained infertility and doesn't know what to do next. Advice sought.", "Wants to know how to improve sperm count naturally" and "Looking for help from others, so he doesn't feel alone".

These codes were then copied to post-it notes and categorised into overarching themes, which were then tagged and imported back into NVivo and combined with the initial codes. The data underwent multiple iterations to ensure the richness of the content was fully explored. The resulting codes were discussed with co-authors and the final topics were iterated and defined. 


\subsection{Ethical Considerations}

The ethical issues of using online "public domain" data in research should be carefully considered as its use may violate the privacy expectations of users. Whilst a user may have posted something in a public forum, certain expectations around how that data will be used exist [48]. Furthermore, it is often not technically or practically possible to contact each user to gain explicit consent.

In developing this study, we considered the ethical implications of using this data, drawing on The British Psychological Society code of ethics [62] and ACM Code of Ethics and Professional Conduct [29] to inform our study development.

In doing so, we evaluated the potential costs to participants, as well as the benefits that using this data may provide to this population. Since informed consent could not be obtained from forum contributors, care was taken to ensure all posts extracted were anonymised by replacing usernames with unique six-digit codes. Only the body of comments were used in analysis. To ensure we do not indirectly reveal the identities of forum users, the forums are never named. Moreover, to reduce the risk of anonymised quotations being deanonymised, each illustrative quotation is paraphrased.

Ethical approval was granted by the UCL Interaction Centre ethics committee.

\section{FINDINGS}

Search terms identified four unique forums. One forum was not compatible with the scraper tool, so we could not readily extract those posts. A total of 902 threads were extracted from three unique forums.

After the data were cleaned in accordance with our study's inclusion and exclusion criteria, 101 threads remained. The number of posts per thread varied (Min $=1, \operatorname{Max}=61)$. In total, $N=603$ posts were included in our analysis. These posts were made in the period from January 2005 to July 2018.

Initial coding of the forum threads resulted in 14 codes. Further iterations of coding resulted in these codes being further reduced into three main themes: (1) Seeking and Providing Advice in Online Communities, (2) Negative Emotions of Internalising Stigma, and (3) A Safe Space Online for Men to Share Anonymously.

\subsection{Seeking and Providing Advice in Online Communities}

Our analysis found the forums were being used by men to share clinical information with an assumed non-clinical population to facilitate advice seeking, health investigations and informal diagnosis. Due to the essential role of sperm in conception for men, different factors relating to sperm quality were common areas of discussion and concern. Additionally, advice was sought in relation to fertility pathways and procedures, as well as medical conditions that may affect fertility. We found men engaging in a form of online camaraderie in their interactions with one another. This appeared to be a social mechanism used to reduce the negative effects of internalised stigma experienced around male-factor infertility, which is further discussed in section 4.2.

4.1.1 Using online communities to interpret clinical advice and results. We found men seeking clarity or confirmation around clinical results and advice received from health professionals. This was commonly focused around sperm quality and semen analysis tests, and posts would often contain detailed medical information. For example, 712533 posted: The doctor said I had $12 \mathrm{million} / \mathrm{ml}$ and the total was 75 million. I was told that concentration should be over 20 million/ml but that the total is good. I don't understand whether or not this is normal. All other parameters are normal. Uncertainty around the boundaries of normality in relation to tests was a regular point of discussion.

Advice from this community of men going through a similar experience appeared to attract increased levels of trust in the information shared, and was highly valued. Clinical advice was 
not being ignored, but instead used to identify similarly positioned men, allowing them to find one another in these online spaces. This enabled more relevant sharing of personal experiences, guidance, and advice, and allowed men to establish what was normal in accordance with these similarly positioned men.

Whilst online forums did not replace clinical expertise, they acted as a mechanism to support men in interpreting clinical advice and results [11], and enabled them to generate informed questions that could be referred back to clinical professionals. Men often used these forums to become localised "experts" around their own condition, affording them an increased perception of control and empowerment over their situation. For example, 722731 sought feedback on the actions of his clinician, : I'm writing on here to see if all of these actions seem normal? What should I do or might I suggest to my urologist?

As well as discussions around semen analysis tests, men with conditions that impair male fertility also engaged on these forums. One commonly discussed condition was Varicocele ${ }^{1}$, with men seeking advice on the medical procedure for rectifying this condition and advice on recovery. They used these forums to equip themselves with expertise and knowledge before having a medical consultation. For example, 458563 posted: my doctor did a varicocele ligation for me and told me to wait for 3-6 months. I'm really worried. Should I do a testicular biopsy or wait as my doctor says? My real concern is the high $\mathrm{FSH}^{2}$. Some research suggests there is no relation between varicocele and fertility. This is hell. Any help?

Men who were seeking or offering advice to others in the forums were often at different stages of their fertility journey. Whilst some had only recently become aware of conception difficulties, others had been experiencing and dealing with the impact of infertility for much longer. For some, clinical interventions such as in-vitro fertilisation (IVF) were no longer viable and other options such as sperm and egg donors were discussed. There was often hesitancy and concern around how people outside these online forum communities would respond to these alternative, non-conventional forms of conception. Online forums provided these men with a space to express themselves and their views around donor conception. They could learn from the experiences of others, gain encouragement and support and ultimately work towards internalising their chosen conception route, before discussing it with others.

Men also used these forums to share information about their partner to gain advice.

It was not uncommon that one member of a couple would be more informed than the other, so men used these online forums to gain knowledge and become informed to support their partner. They appeared to use the online forum to find others who understood their situations. This ranged from trying to become an "expert" around their condition, to seeking advice on how to support their partner emotionally; for example, 435034 shared: My tests are clear and the issue is with her tubes. I understand her thoughts of "it's her body, her issues, her grief" but it's driving us apart. What is my role? Do I just keep quiet or how can help her see I'm there to support and take an active role?. They were not only seeking to take control of their situation: there was a sense of re-establishing their role within the relationship. For example, 484042 posted: I am learning slowly but surely and I want to know more so that I can be the best husband possible. Such posts often received responses including encouragement and insights from knowledgeable others who would willingly reply to posts that requested advice and information based on their experience. Men also frequently spoke about the emotional impacts of feeling unable to support their partner, which we discuss in more detail in Section 4.2. This further highlights the benefit of online forums: to share previous experiences to support one another.

\footnotetext{
${ }^{1}$ Varicocele is a condition in which the veins in the testicle are abnormally enlarged and can affect fertility.

${ }^{2}$ FSH: Follicle Stimulating Hormone, a reproductive health related hormone.
} 
4.1.2 Attempting to Understand the Cause. It is not uncommon for issues around fertility to be unexplained [17]. Our analysis identified the difficulty men found themselves in with managing this uncertainty. Therefore, one of the investigative uses of these forums was exploring the cause of their fertility issues. Men would discuss previous medical conditions, or drugs taken, often recalling events that happened many years previously. For instance, 726580 posted: I read previous research about side effects of Propecia. I'm afraid my short-term use about 10 years ago might affect my semen count or quality now. Is there any real reason to be concerned?

Some of the discussions that occurred around causes of infertility focused on the need to validate men's pre-existing ideas on what was preventing them conceiving naturally. These discussions often led others to provide reassurance that they were not to blame, attempting to alleviate guilt that may have developed through being unable to provide a child for their partners. 726288 posted: You have no control over it [infertility], and you did nothing wrong, so really, try to dismiss that feeling [of guilt].

We found that men provided comfort to one another in cases of unexplained infertility, through the sharing of personal experiences and reassuring support. This enabled men to place themselves with similar others and provided a sense of comfort.

4.1.3 Seeking and sharing practical steps for improving fertility. As well as sharing advice around clinical information, we found men shared advice around lifestyle, vitamins and supplements to help improve fertility. This advice was often quite specific, with the inclusion of precise doses, supported by personalised stories of how these strategies had improved their own sperm quality, and in some cases, resulted in successful conception. This acted as evidence of the credibility of the advice, and appeared to encourage those seeking these recommendations.

In summary, we found online forums being used to ask often very detailed and private questions related to reproductive health and clinical advice once men experienced a disruption of their assumed fertility. These online spaces provided men with an easily accessible community of similarly positioned men in which they felt comfortable to discuss their individual concerns, seek comfort and make sense of their experiences. Men used the information and advice gained to support themselves in their interactions with medical professionals, explore ways to support their female partner and gain an understanding of their new and unexpected fertility circumstance.

\subsection{Negative Emotions of Internalising Stigma}

Across all of the forum threads analysed, there were many conversations that intersect across the topics reported in Section 4.1 that also centred around men's emotional well-being. When looking for clinical advice, support on interpreting diagnoses and sharing advice on how to improve their fertility, users were also expressing a sense of internalised stigma and were looking for a source of emotional support on how to handle their situation or circumstances. Conversations that began with the sharing of clinical information would often switch to a request for support or to find others who had had similar experiences. Men turned to online forums as a space to share their negative emotions, as expressed by 713715: Who [else] do we turn to for support? We carry the burdens of a family. We do so gracefully, but not without internalising the pain of failure.

Through the sharing of men's intimate fertility journeys, we can see the effect the process had on their sense of masculinity. The feeling of responsibility contributed to their feelings of fear and anxiety, that in turn threatened their ability to access healthcare or to turn to others to converse and share with.

There was an assumed role that men supposed themselves to have, get my wife pregnant, which was shared by a number of men on the forum. They felt as if they were failing in their role within the couple, as well as primitively as a man. As shared by 301338: I feel like I am less of a man since I 
cannot get my wife pregnant and provide her with a family... I can't let her down by being weak. I give my wife everything she desires, but I have already let her down by not being able to give her the one thing she desperately wants: a baby

Others in the forum would often reply with empathy at having felt similarly as well as with encouragement that you may in fact be more of a man for going the extra mile to achieve your goals of becoming a family. Forum users aimed to reduce the internalised stigma felt and shared by others by providing assurance and encouragement, and sharing views and feelings with one another. Other men in the forum would exhibit a sense of camaraderie and seek to reduce the threats to masculinity that users shared by exhibiting acceptance of perceived emotional weaknesses. We did not find evidence in the data of users discouraging one another or replying with negativity. The fact that users are supportive and empathetic seems to be a universal characteristic of the online community of men who have experienced fertility difficulties.

Men's anguish at encountering difficulties conceiving appeared to have a great impact on their emotional well-being and their perceived masculinity. This included feelings of guilt, which they had to come to terms with, that even if the fertility issue was male-factor, their female partner was the one who had to go through invasive and sometimes painful medical procedures.

Many of these emotions were externalised on the forums, with posts portraying anger, frustration, and feelings of uselessness and defectiveness: all signs of developed internalised stigma. As poignantly shared by 725618: It's hard to tell the world that I feel like only half a man... I don't understand why my wife hasn't left me when all she wants is a child.

Men often discussed feeling unable to show their emotions to their partner. Men would describe the pain and anguish their partners were feeling but they themselves did not want to add to this by externalising their emotions to their partner. Online forums provided men with the opportunity to express their emotions in an environment where they could benefit from support and understanding from other users, without the perception of burdening their partner, or risking being negatively judged.

Experiencing difficulties conceiving was described as the worst experience by some of the men in the forums. Threats to perceived masculinity were evident in the use of language around fertility. Whilst the experience of managing fertility is commonly referred to as a "journey", here we found men describing it as a lonely "battle" or "war" that needed to be won. Users often reported feeling mentally unprepared for the emotional strain that they felt, or the impact it had on their relationships. There were frequent mentions of related relationship problems. These ranged from wanting to be there for their partner, not sharing that they were also struggling and the increase in hostility and lack of patience towards one another which negatively affected the relationship. Forum posts often disclosed that there was a feeling of detachment or disconnection in the relationship that led them to the online forum.

Men expressed difficulties towards "The pain in sharing other people's joy". This referred to having to manage their own and their partner's emotions when hearing announcements of pregnancies from people in their physical network and community. There is a difficulty in trying to manage the internal conflict of being happy for their friends and the pain they feel of not being able to be in the same position. As shared by 301338: It's tough to sit and see so many people are enjoying life with children. These comments were often followed up with pleas to understand how others dealt with these difficult emotions. This was particularly difficult for couples who had been struggling for an extended period of time.

Men turned to online forums for a safe space to vent, share and get advice on how to manage these internalised emotions and their partner's feelings. 301338 wrote: I have to be positive. I can't let my wife down [...] Anyway, thanks for letting me vent. I needed it 
Positive emotions were also shared, but less often. Users were more likely to share encouragement and positive support for others, rather than initiating posts with optimism unless an active user was providing an update to a previously shared story.

In summary, the majority of the users on these forums were experiencing reproductive difficulty and used online forums for emotional support, factual information and practical guidance to help with sense-making and meaning-making. This included managing senses of guilt, anger and weakness; they expressed feelings of being alone while seeking support from other people who could empathise through shared experiences. Online forums were used as spaces to share intimate and negative feelings as they brought together an identified community of users who have undergone similar experiences. Experiences from others were then used to bridge the gaps in knowledge to manage their fertility journey and make sense of their new circumstances. Threads expose internalised stigma felt and shared through detailed tales of anguish and despair. We find evidence of forum users finding comfort in knowing that they are not the only ones experiencing such misery.

\subsection{A Safe Space Online for Men to Share Anonymously}

We found online forums acted as a "safe space" for men to vent frustrations and share their innermost personal feelings without fear of judgement or negative social consequence. Men would often share personal stories that they would not, or felt that they could not, share with others outside the forum. Although the fertility journey was one a couple usually embarked upon together, there was a sense of isolation felt from their friends, families and co-workers. This was shared by 725845: Are there any other men like me in the same situation? I feel really alone on this journey. How do you cope?

As well as using forums to provide information or advice, or to explicitly seek emotional support, men found them to be an accessible place to connect with others who were similar to them in terms of experience or situation and to share their fertility journeys with others who could empathise (rather than just sympathise). They formed an online community where they shared intimate stories and supported one another to manage their unexpected fertility journey. Men specifically sought to hear from men who had completed their fertility journey and had success stories to share, as well as advice on how to cope with a "new normal" of life without biological children.

Men stated that they could not talk to others or did not have an audience that would be as understanding, attentive and supportive, and chose the online forum method of collaborative communication, sometimes over face-to-face interactions with people in their physical network, as shared by 435034: I don't know any other men I can discuss this with so I came here.

This was particularly heightened if men felt that they could not speak to or be open and honest with their partner. This could be for a range of reasons from not wanting to burden them, feeling guilty towards them or not feeling like they had an empathetic audience in their partner, as detailed in section 4.2. Being part of such an online forum made them feel part of a community and less abnormal. I joined this group because I'm trying to make sure I'm not alone in this situation. (724865)

These online forums were spaces where users could communicate with one another in an understanding and helpful manner. There was often expression of appreciation for others' time, advice and kindness. For some, it was clearly important to engage with (and receive responses from) other men (rather than women), as shared by 579641: we men like to discuss things amongst ourselves and No offence to women our there, but sometimes men do prefer to talk about things among ourselves before we ask for help from women.

Online forums allow users to hold anonymous conversations and support one another through the fertility journey. They also enable people to lurk (to read without necessarily posting). The likely presence of lurkers is indicated by the number of views on a thread compared with the number of posts or replies on the same thread: some of the discrepancy between these figures 
can be accounted for by lurkers; some may also be due to users following a thread that they have previously contributed to. Some users identified themselves as previous lurkers, such as 579641 in his initial posts in the online forum: I am new to this forum, but I have been reading quite a bit using my wife's ID. I decided to sign up so that I could talk to the husbands out there, to relate with them and also trade experiences.

This illustrates how some men chose to join the conversations publicly, having learned what the forums are about and what the social norms about interacting are. While lurkers might not actively contribute to the community, they receive support from it, and may become contributors over time.

Overall it was found that men who use online forums whilst experiencing fertility difficulties are looking for very personal and specific information and advice on how to manage their fertility journey and improve their chances of being able to conceive. They were also used to gather information to bridge knowledge gaps and understand what was normal within this socially constructed community. It was important for men to hear from other men who were going through a similar journey or had done so in the past to provide first-hand advice. This was seen from the level of personal information that are shared in posts as well as the gratitude expressed upon receiving encouraging advice and support. Online forums were also used as safe spaces to share their feelings and experiences in order to confirm that they were not an anomaly or alone in their thoughts and feelings. Comparing themselves to others in these online forums also appeared to bring a sense of comfort, which they may feel unable to attain in their offline networks. Men were able to share their intimate feelings, which often indicated a sense of internalised stigma, which could lead to feelings of insecurity and pose a threat to their perceived masculinity.

\section{DISCUSSION}

This analysis of online forum comments shows how some men use online spaces to openly discuss their thoughts and feelings and to share their experiences with empathetic anonymous audiences. We found that users sought to connect with one another in order to express themselves in a safe space and help them understand their "new normal".

Users shared their weaknesses and desires with one another and received support, guidance, and advice on how to progress towards their parenthood goals and manage their fertility difficulties. Inherent social norms in these online forums mean that they provide anonymised spaces where people can share information and feelings towards a wide range of topics without fear of judgement and with the expectation of support. This has also been found in work by Ziebland et al. [68], who found that users would seek opinions, support and help in interpreting clinical information with anonymous inquiries.

Through the analysis of 603 online forum posts, we found that men used these online forums to look for tailored information and advice, and to engage with peers for emotional support. In many cases men shared that they did not have another trusted outlet or community to share with. The themes we present in our findings are interrelated; we use the model of finding a "new normal" [27] to discuss these themes in more detail, and to unpack and explore the relationships between them.

\subsection{Engaging Online with Others to Find a "New Normal"}

We found that men used the forums for support in their information seeking journey to obtain advice on how to manage their fertility concerns and conception difficulties in their daily lives, which can be interpreted as the need to find their "new normal". Genuis and Bronstein [27] highlight the importance of finding information and validating thoughts and knowledge with others; this was also important to men using these online forums. Also discussed by others, (e.g., [49]), the need to seek information can contribute to the understanding of what is normal for them (men), their condition (infertility) and what is normal for peers who are similar to them (forum users).

Proc. ACM Hum.-Comput. Interact., Vol. 3, No. CSCW, Article 1. Publication date: November 2019. 


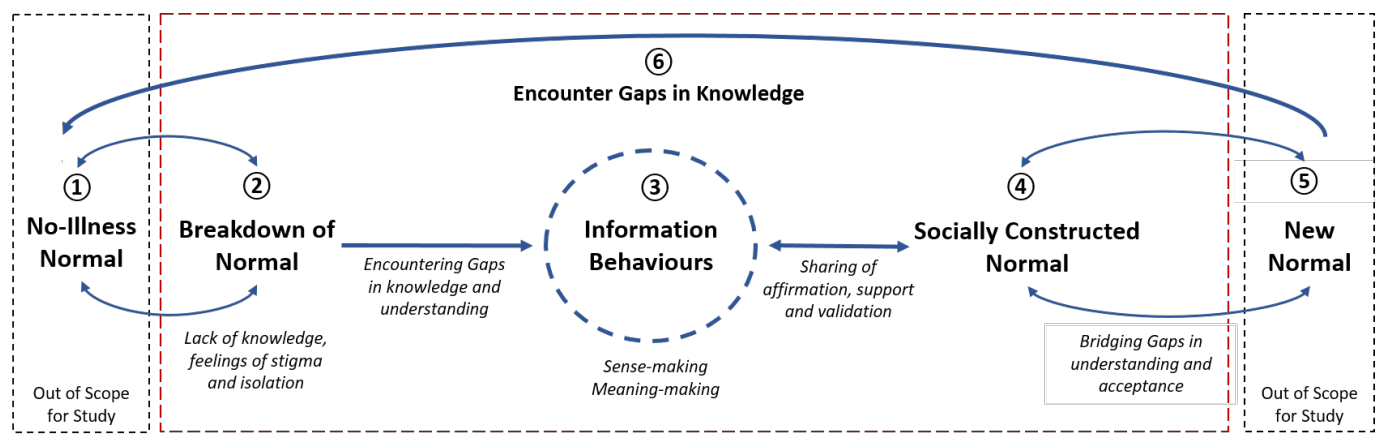

Fig. 1. Finding New Normal Model [27] elaborated to the context of Fertility

Our findings show us how some men use online forums to transition from experiencing a life disruption by engaging in sense-making and meaning-making towards understanding what a socially constructed normal can look like for them. This enables us to elaborate Genuis and Bronstein's model of finding a "new normal", contextualised to the situation of using online forums as a resource for coming to terms with a stigmatised condition. This is illustrated in Figure 1 and discussed below.

(1) Men who have no particular or recognised fertility concern do not seem to engage with the sampled online forums; consequently, the "no illness normal" phase is not well represented in our forum data and is out of scope for this study.

(2) Initially, when people become aware of their fertility difficulties (whether through experiencing difficulty in conceiving or through a formal diagnosis), their sense of what is normal is challenged. This breakdown is exacerbated by the difficulty of discussing either the condition or the experience due to stigma, particularly as experienced by men. This, in turn, is partly a function of infertility being a stigmatised condition that is not widely discussed. There can be a lack of knowledge in this phase, which leads men to recognise the need for information and support.

(3) Men engage in information seeking and sense-making through various channels, often with their partners and clinicians, and through online media. Many forum participants reported finding it difficult to discuss issues with others in their social or personal circles, so the forums were an important source of information. Men solicited (and received) factual, often clinical, information; conversely, in these online social networks, men also shared information - both about themselves, and also about their understanding of clinical conditions. This enabled men to interpret and validate information from other sources and engage in meaning-making, which is internalising the information found and exploring what that may mean to their individual situation [56].

Information seeking from online forums, as well as discussions peers may be engaged with on the forums, can also lead to participants identifying further gaps in their understanding. They may recognise a need for information and guidance on what they can do to take control or impact the situation in a positive manner. We postulate that this information is either sought from the online forum community or from other sources and finally validated and interpreted through the forum as shown at point (6) of Figure 1. 
(4) As well as exchanging and interpreting factual information, men also solicited and received emotional support, affirming the normality of the situation by sharing with peers. This was done in a number of ways including sharing experiences, and using language to manage uncertainty and to bring comfort and acceptance [6]. This meant that the social groups (within the online forums) constructed and maintained their localised sense of normal within these online communities of men. The stigma around the condition limited the social construction of normal in people's everyday lives.

(5) The socially constructed normal within these online communities could have enabled individuals engaging within these communities to come to terms with, and take ownership of, their situation (e.g. in engaging in their fertility journey and relating with their partners). Support within these online communities enables at least some of the participants to manage better within their day-to-day lives. In other cases, notably when couples conceive successfully, it is possible to move on to a (different) no-illness normal, related to fatherhood; this is out of scope in our current study.

(6) As men engage in their fertility journey, and as understanding develops and experiences change, new discontinuities or gaps in understanding can be encountered. In some cases, these lead to a further refinement of their sense of what is normal being negotiated within (and outside) the forums.

Some of our findings are reflected by others; for example, Hanna and Gough [34] report how some men use online forums to seek information to "normalise help-seeking". Malik and Coulson [44] found online forums were used to make sense of infertility experiences and reduce feelings of isolation. These findings were echoed and extended in our study, which showed that men were often inquiring whether their situation was normal in relation to other forum users and seeking reassurance from users who had experienced similar circumstances and outcomes.

The behaviour of comparing themselves with other users can be seen as another means of assessing their "new normal", which is different from their existing perceptions. Through the enquiries shared by men to identify how others deal with stigmatising experiences and feelings, we find that users attempt to identify a socially constructed normal (within the online community) and place themselves within it.

Therefore, we find that the processes involved in finding a "new normal" for men who are experiencing difficulties when trying to conceive are reflected in the ways men use online forums during this time; this is illustrated in our elaborated model of finding a "new normal" in Figure 1.

\subsection{Online Resources as a Parallel Source of Health Information, alongside Clinical Expertise}

When Porter and Bhattacharya [51] studied couples undergoing fertility treatment, they identified how dissatisfaction with information provided by health professionals would often lead to information-seeking from other sources. We also found some men being dissatisfied with information provided to them by their health professionals. When information from partners was also deemed unsatisfactory, they used online forums as a source of non-clinical advisers. This allowed them to seek information and advice to help them normalise their situation.

We found that when users were looking for information or advice, they would provide background information to enable other forum users to become familiar with their situation, then ask specific questions. These could consist of "what" and "how can I" questions in order to seek specific information or advice to improve their current situation. Other studies on online health information 
have also identified this type of behaviour (e.g., [14]); we found that active forum users addressed questions around preconception health and infertility by providing advice and opinions generously.

Maltz and Borker [45] report that men are more likely to want to state and share facts and opinions than women. In forums when this type of information was requested, other users commonly obliged. However, we found that the support provided in the forums we analysed went beyond the sharing of facts and opinions, with users providing encouragement and empathy to help reduce the internalised stigma felt by others.

Previous researchers have highlighted the need to validate online advice or information to ensure that information is accurate (e.g., [46]). However, there are no obvious checks on these public forums to ensure information shared is factually correct, apart from other users or forum moderators commenting on information provided. It was rare for other users to obviously "moderate" or correct one another. The norms of engagement in the forums analysed in this study promoted engagement with peers and the rapid development of trust between community participants, as discussed in section 5.3.

Valuable roles for forums such as the ones included in this study include helping men to make sense of the situations they find themselves in, as other people with similar experiences help with interpreting information and with making the situation seem more normal. While this general value of online forums is widely recognised, this study has highlighted the particular kinds of information that were of concern to men experiencing infertility who used online forums. There is also a conspicuous absence of discussion of preconception health unrelated to fertility concerns in these forums, despite this being a significant factor in fertility and subsequent child health [65]. It seems that once men's "normal" is disrupted, these forums become a potential resource for information-seeking, but do not attract men in the "no illness normal" fertility phase when couples are trying to conceive naturally.

\subsection{Seeking Validation through Emotional Engagement with Supportive Peers Online}

As many questions raised by men could have arguably been answered using online search engines or conversations with their health professionals, there is a question about why these users chose online forums to share and seek information rather than (or supplementary to) other, more authoritative sources of information.

One reason may be to support sense-making (i.e. information interpretation), to make information personally meaningful. This can be seen through the sharing of their sensitive personal information, followed by the request for advice or information as well as an ensuing dialogue. Standard information resources, however authoritative, do not directly support interpretation. Trust is maintained through the social norms developed within the online forums, and through the public scrutiny of all posts. There are benefits to collaborative forms of information seeking [58], specifically around information that may be harder to find such as fertility advice for specific conditions and experiences.

Health professionals also support information interpretation but, as previous studies have reported, both men and women often find interactions with medical professionals difficult and insensitive, and men often feel left out of the conversations, as medical professionals communicate primarily with the female partner (e.g. [7]). In online forums, men can relate to others as equals, and other men give their time freely to engage.

Another unique quality of online forums is the shared peer community who provide a safe space with no judgement for people who use them [53]. Whilst men are likely to feel hesitation to seek help and expose their emotional vulnerabilities, the anonymity of online forums encouraged men to share their feelings [38]. This has also been found in studies of gender transition [31], where similar people were more likely to openly share and seek emotional support from one another. The safe space is 
also reinforced through the use of non-identifiable user names to preserve the user's anonymity. This can enable users to openly share intimate details and have open conversations [5] in order to obtain the benefits of accessing social support without the fear of judgement or embarrassment. It has been noted by Ammari et al. [4] that men can fear stigma and judgement if they openly share online, so the property of an assumed safe space is vital for hidden and marginalised populations to discuss sensitive topics.

We found that even in posts that focused on seeking advice, the conversation would often shift into identifying others in a similar position and comparing experiences and understanding with one another, seeking validation and reassurance. When seeking validation of their actions or decisions, men would share information or advice they had been given, then ask other forum users whether they agreed, to satisfy themselves that they should follow the advice. Men seeking validation also benefited from evidence based factual information that helped to reassure them about their interpretations regarding their own situations. Men benefit from using online forums to obtain this type of information, as it is a space where they can compare their understanding with that of others [18].

In some cases, the users are very specific that they want to hear from other men and not women. This was also found by Richard et al. [54], who report that men were less responsive to women who replied to them than to men. These authors specifically report that men used online infertility discussion boards to "acquire support from similar others."

Others (e.g., [44]) have discussed the value of forums to couples seeking fertility treatments, and for men (e.g., [38]) dealing with sensitive health topics. Hanna and Gough [34] report on men's use of online forums, focusing particularly on the emotions expressed. Our study supports their findings, further highlighting the close inter-relationships between seeking practical advice and information and seeking emotional support and assurances that are contextualised to their specific issues and situations.

\section{LIMITATIONS AND FUTURE WORK}

It is likely that there is an inherent selection bias in our sample of forum users in comparison to the general population of men preparing for conception or facing infertility. Forum users are more likely to be open and share personal and sensitive information about themselves and their partners. There may be other concerns in the wider population of men who experience fertility difficulties. This has been found in other studies which explored forum use [40]. However, it has been reported that using online methods of data collection does not invalidate findings, especially when investigating a perceived stigmatised or sensitive topic [41].

Whilst our analysis of online forum data aimed to explore how men use online forums to communicate with one another when they experience infertility, we are unable to identify the needs and requirements of "lurkers", a hidden group of forum users. The phenomenon of hidden users was reported by Panciera et al. [50] who discuss the difficulties of giving a voice to lurkers as legitimate users.

Due to the data gathering method, we are unable to probe the users to explore their feelings or the intentions behind their posts. In particular, this study does not enable us to probe apparent paradoxes in the ways that men used these forums: that there is apparent intimacy with strangers; that people place their trust in anonymous others; and that very personal information is shared in a public space. These themes are explored by Hanna and Gough [34], who highlight the centrality of anonymity in making such online forums function.

We are also unable to explore the implications of cultural background or experiences of the users who post on these online forums in order to ascertain the influence this may have on their decision to share and what they choose to share on anonymous online forums. We believe that, given

Proc. ACM Hum.-Comput. Interact., Vol. 3, No. CSCW, Article 1. Publication date: November 2019. 
the topic of research, participants' rights to anonymity and confidentiality override the potential benefits this line of enquiry would add to our presented contributions.

In future work, we propose to engage directly with men facing fertility challenges, to identify their needs and experiences that are not addressed through online forums, and characterise their information journeys. We will also investigate the views of men who are either lurkers or who experience infertility but do not use online forums, to better understand the existing ecology of digital and physical resources to support men and identify opportunities for future interventions.

As men may use online forums as sources of information instead of or in parallel to their health professionals, further work could also consider the implications of using online forums or social network sites as a suggested health peer-led resource for men who are trying to conceive. There could also be a beneficial impact to connecting men who experience infertility and do not currently use online forums with like-minded anonymous online communities for social support.

Further work could also seek to understand aspects of the elaborated finding "new normal" model we have presented that cannot be understood using an online forum population. In particular, this might investigate the information seeking behaviours of men when they are in the "no-illness normal" fertility stage and the final "new normal" stage that men who experience fertility difficulties may adopt outside the online forum.

\section{CONCLUSIONS}

In this study we found that men are likely to use online forums when they encounter fertility difficulties when trying to conceive, but found little evidence of them being used to discuss general preconception health in the absence of concerns about infertility. The analysis of forum posts identified that men used online forums to look for information and health related advice about sperm health, investigations and diagnoses, and that the search for information is woven into discussions in which men also seek validation of decisions and reassurance that they are not alone whilst they are experiencing difficulties in trying to conceive.

Men used online forums as safe spaces to discuss and share their feelings of internalised stigma with a community of like-minded people who could empathise and support them during this unanticipated emotionally difficult time. There is a need for men who are experiencing difficulties whilst trying to conceive to establish a "new normal", often in relation to other men who are undergoing similar experiences. This enables them to understand the process and reduce self-blame caused by the internalised stigma.

Online forums were used as a resource to bridge gaps in understanding once men experience the life disruption of infertility. Online users support sense-making and meaning-making in order to establish a sense of socially constructed "new normal" with their peers in the online community. Conversations were held between groups of like-minded men, which led to the normalisation of men's involvement into the fertility journey, which could contribute to reducing the societal pressures of unequal gender balance in the domain of fertility.

The study reported here extends our understanding of how men communicate with one another online when they experience fertility issues and of the unique role online forums play in facilitating connections, finding a "new normal" and providing a platform for men to discuss private, sensitive and sometimes stigmatised issues behind a screen of anonymity in a public online space.

\section{ACKNOWLEDGEMENTS}

This research is partly funded by EPSRC Studentship and by the EU Horizon 2020 research and innovation program under the Marie Skłodowska-Curie Action ITN grant agreement No 675730 and the University College London (UCL) Department of Computer Science. 


\section{REFERENCES}

[1] Ashok Agarwal, Aditi Mulgund, Alaa Hamada, and Michelle Renee Chyatte. 2015. A unique view on male infertility around the globe. Reproductive biology and endocrinology 13, 1 (2015), 37.

[2] Tawfiq Ammari and Sarita Schoenebeck. 2015. Understanding and Supporting Fathers and Fatherhood on Social Media Sites. In Proceedings of the 33rd Annual ACM Conference on Human Factors in Computing Systems (CHI '15). ACM, New York, NY, USA, 1905-1914. https://doi.org/10.1145/2702123.2702205

[3] Tawfiq Ammari and Sarita Schoenebeck. 2016. Thanks for your interest in our Facebook group, but it's only for dads: Social Roles of Stay-at-Home Dads. In Proceedings of the 19th ACM Conference on Computer-Supported Cooperative Work \& Social Computing. ACM, San Francisco, California, USA, 1363-1375.

[4] Tawfiq Ammari, Sarita Schoenebeck, and Silvia Lindtner. 2017. The Crafting of DIY Fatherhood. In Proceedings of the 2017 ACM Conference on Computer Supported Cooperative Work and Social Computing. ACM, Portland, Oregon, USA 1109-1122.

[5] Tawfiq Ammari, Sarita Schoenebeck, and Daniel M Romero. 2018. Pseudonymous parents: Comparing parenting roles and identities on the Mommit and Daddit subreddits. In Proceedings of the 2018 CHI Conference on Human Factors in Computing Systems. ACM, 489.

[6] Lynda Appleton and Maria Flynn. 2014. Searching for the new normal: Exploring the role of language and metaphors in becoming a cancer survivor. European fournal of Oncology Nursing 18, 4 (2014), 378-384.

[7] Shafali T Arya and Bridget Dibb. 2016. The experience of infertility treatment: the male perspective. Hum Fertil (Camb) 19, 4 (2016), 242-248.

[8] Magdalena Berger, Todd H Wagner, and Laurence C Baker. 2005. Internet use and stigmatized illness. Social science \& medicine 61, 8 (2005), 1821-1827.

[9] Paul Best, Elena Gil-Rodriguez, Roger Manktelow, and Brian J Taylor. 2016. Seeking help from everyone and no-one: Conceptualizing the online help-seeking process among adolescent males. Qualitative health research 26, 8 (2016), 1067-1077.

[10] Lindsay Blackwell, Jean Hardy, Tawfiq Ammari, Tiffany Veinot, Cliff Lampe, and Sarita Schoenebeck. 2016. LGBT parents and social media: Advocacy, privacy, and disclosure during shifting social movements. In Proceedings of the 2016 CHI conference on human factors in computing systems. ACM, 610-622.

[11] Ann Blandford and Simon Attfield. 2010. Interacting with information. Synthesis Lectures on Human-Centered Informatics 3, 1 (2010), 1-99.

[12] Virginia Braun and Victoria Clarke. 2006. Using thematic analysis in psychology. Qualitative research in psychology 3 , 2 (2006), 77-101.

[13] Eleanor R Burgess, Madhu C Reddy, Andrew Davenport, Paul Laboi, and Ann Blandford. 2019. "Tricky to get your head around": Information Work of People Managing Chronic Kidney Disease in the UK. In Proceedings of the 2019 CHI Conference on Human Factors in Computing Systems. ACM, 665.

[14] Adrian Bussone, Simone Stumpf, and Stephanie Wilson. 2017. The use of online forums by people living with HIV for help in understanding personal health information. International journal of medical informatics 108 (2017), 64-70.

[15] Alison Chapple, Sue Ziebland, and Ann McPherson. 2004. Stigma, shame, and blame experienced by patients with lung cancer: qualitative study. BMJ 328, 7454 (2004), 1470.

[16] Constantinos K Coursaris and Ming Liu. 2009. An analysis of social support exchanges in online HIV/AIDS self-help groups. Computers in Human Behavior 25, 4 (2009), 911-918.

[17] Tara M Cousineau and Alice D Domar. 2007. Psychological impact of infertility. Best Practice \& Research Clinical Obstetrics \& Gynaecology 21, 2 (2007), 293-308

[18] Munmun De Choudhury, Sanket S Sharma, Tomaz Logar, Wouter Eekhout, and René Clausen Nielsen. 2017. Gender and cross-cultural differences in social media disclosures of mental illness. In Proceedings of the 2017 ACM conference on computer supported cooperative work and social computing. ACM, 353-369.

[19] Sarah Denford, Diana Harcourt, L Rubin, and A Pusic. 2011. Understanding normality: a qualitative analysis of breast cancer patients concepts of normality after mastectomy and reconstructive surgery. Psycho-Oncology 20, 5 (2011), 553-558.

[20] Suzanne S Dickerson, Amber Reinhart, Marcia Boemhke, and Laila Akhu-Zaheya. 2011. Cancer as a problem to be solved: internet use and provider communication by men with cancer. CIN: Computers, Informatics, Nursing 29, 7 (2011), 388-395.

[21] Maeve Dooley, Aonghus Nolan, and K M Sarma. 2011. The psychological impact of male factor infertility and fertility treatment on men: a qualitative study. The Irish fournal of Psychology 32, 1-2 (2011), 14-24.

[22] Louise A Ellis, Philippa Collin, Patrick J Hurley, Tracey A Davenport, Jane M Burns, and Ian B Hickie. 2013. Young men's attitudes and behaviour in relation to mental health and technology: implications for the development of online mental health services. BMC Psychiatry 13, 1 (2013), 119. 
[23] Mayara Costa Figueiredo, Clara Caldeira, Tera L Reynolds, Sean Victory, Kai Zheng, and Yunan Chen. 2017. SelfTracking for Fertility Care: Collaborative Support for a Highly Personalized Problem. Proceedings of the ACM on Human-Computer Interaction 1, CSCW (2017), 1-21.

[24] Jane R W Fisher, Gordon H W Baker, and Karin Hammarberg. 2009. Long-term health, well-being, life satisfaction, and attitudes toward parenthood in men diagnosed as infertile: challenges to gender stereotypes and implications for practice. Fertility and Sterility 94, 2 (2009), 574-580.

[25] Keith A Frey, Shannon M Navarro, Milton Kotelchuck, and Michael C Lu. 2008. The clinical content of preconception care: preconception care for men. American Journal of Obstetrics and Gynecology 199, 6 (2008), S389-S395.

[26] Kenneth Gannon, Lesley Glover, and Paul Abel. 2004. Masculinity, infertility, stigma and media reports. Social Science \& Medicine 59, 6 (2004), 1169-1175.

[27] Shelagh K Genuis and Jenny Bronstein. 2017. Looking for "normal": Sense making in the context of health disruption. fournal of the Association for Information Science and Technology 68, 3 (2017), 750-761.

[28] Erving Goffman. 2009. Stigma: Notes on the management of spoiled identity. Simon and Schuster.

[29] DW Gotterbarn, Bo Brinkman, Catherine Flick, Michael S Kirkpatrick, Keith Miller, Kate Vazansky, and Marty J Wolf. 2018. ACM Code of Ethics and Professional Conduct. (2018).

[30] Xinning Gui, Yu Chen, Yubo Kou, Katie Pine, and Yunan Chen. 2017. Investigating Support Seeking from Peers for Pregnancy in Online Health Communities. Proceedings of the ACM on Human-Computer Interaction 1, CSCW (2017), 50.

[31] Oliver L Haimson, Anne E Bowser, Edward F Melcer, and Elizabeth F Churchill. 2015. Online inspiration and exploration for identity reinvention. In Proceedings of the 33rd Annual ACM Conference on Human Factors in Computing Systems. ACM, 3809-3818.

[32] Esmée Hanna and Brendan Gough. 2015. Experiencing male infertility: A review of the qualitative research literature. Sage Open 5, 4 (2015), 2158244015610319.

[33] Esmee Hanna and Brendan Gough. 2016. Emoting infertility online: A qualitative analysis of men's forum posts. Health: 20, 4 (2016), 363-382.

[34] Esmée Hanna and Brendan Gough. 2016. Searching for help online: An analysis of peer-to-peer posts on a male-only infertility forum. Fournal of Health Psychology (2016), 1359105316644038.

[35] Melissa Henry, Angela Ho, Sylvie D Lambert, Franco A Carnevale, Brian Greenfield, Christina MacDonald, Alex Mlynarek, Anthony Zeitouni, Zeev Rosberger, Michael Hier, et al. 2014. Looking beyond disfigurement: The experience of patients with head and neck cancer. Fournal of Palliative Care 30, 1 (2014), 5-15.

[36] Lisa Hinton, Jennifer J Kurinczuk, and Sue Ziebland. 2010. Infertility; isolation and the Internet: A qualitative interview study. Patient Education and Counseling 81, 3 (2010), 436-441.

[37] Milton Kotelchuck and Michael Lu. 2017. Father's role in preconception health. Maternal and Child Health fournal 21, 11 (2017), 2025-2039.

[38] Marilynn Larkin. 2001. Men open up in online forums. The Lancet 357, 9254 (2001), 487.

[39] Maria Lohan, Áine Aventin, Han Christina S Oliffe, John L, and Joan L Bottorff. 2015. Knowledge translation in men's health research: Development and delivery of content for use online. Fournal of Medical Internet Research 17, 1 (2015), e31.

[40] Diana MacLean, Sonal Gupta, Anna Lembke, Christopher Manning, and Jeffrey Heer. 2015. Forum77: An analysis of an online health forum dedicated to addiction recovery. In Proceedings of the 18th ACM Conference on Computer Supported Cooperative Work \& Social Computing. ACM, 1511-1526.

[41] Juan F Maestre, Elizabeth V Eikey, Mark Warner, Svetlana Yarosh, Jessica Pater, Maia Jacobs, Gabriela Marcu, and Patrick C Shih. 2018. Conducting Research with Stigmatized Populations: Practices, Challenges, and Lessons Learned. In Companion of the 2018 ACM Conference on Computer Supported Cooperative Work and Social Computing. ACM, Jersey City, NJ, USA, 385-392.

[42] Juan F Maestre, Haley MacLeod, Ciabhan L Connelly, Julia C Dunbar, Jordan Beck, Katie A Siek, and Patrick C Shih. 2018. Defining through expansion: conducting asynchronous remote communities (arc) research with stigmatized groups. In Proceedings of the 2018 CHI Conference on Human Factors in Computing Systems. ACM, 557.

[43] Sumaira H Malik and Neil Coulson. 2008. The male experience of infertility: a thematic analysis of an online infertility support group bulletin board. fournal of Reproductive and Infant Psychology 26, 1 (2008), 18-30.

[44] Sumaira H Malik and Neil S Coulson. 2010. Coping with infertility online: an examination of self-help mechanisms in an online infertility support group. Patient Education and Counseling 81, 2 (2010), 315-318.

[45] Daniel N Maltz and Ruth A Borker. 1982. A cultural approach to male-female miscommunication. A cultural approach to interpersonal communication: Essential readings (1982), 168-185.

[46] Jennifer Mankoff, Kateryna Kuksenok, Sara Kiesler, Jennifer A Rode, and Kelly Waldman. 2011. Competing online viewpoints and models of chronic illness. In Proceedings of the SIGCHI Conference on Human Factors in Computing Systems. ACM, 589-598. 
[47] Michael Massimi, Jill P Dimond, and Christopher A Le Dantec. 2012. Finding a new normal: the role of technology in life disruptions. In Proceedings of the acm 2012 conference on computer supported cooperative work. ACM, 719-728.

[48] Helen Nissenbaum. 2009. Privacy in context: Technology, policy, and the integrity of social life. Stanford University Press.

[49] Aisling Ann O’Kane, Sun Young Park, Helena Mentis, Ann Blandford, and Yunan Chen. 2016. Turning to peers: integrating understanding of the self, the condition, and others' experiences in making sense of complex chronic conditions. Computer Supported Cooperative Work (CSCW) 25, 6 (2016), 477-501.

[50] Katherine Panciera, Reid Priedhorsky, Thomas Erickson, and Loren Terveen. 2010. Lurking? cyclopaths?: a quantitative lifecycle analysis of user behavior in a geowiki. In Proceedings of the SIGCHI Conference on Human Factors in Computing Systems. ACM, 1917-1926.

[51] Maureen Porter and Siladitya Bhattacharya. 2008. Helping themselves to get pregnant: a qualitative longitudinal study on the information-seeking behaviour of infertile couples. Human Reproduction 23, 3 (2008), 567-572.

[52] Magali Prost, Béatrice Cahour, and Françoise Détienne. 2010. Analysing online social support between professionals. In Proceedings of the 28th Annual European Conference on Cognitive Ergonomics. ACM, 341-342.

[53] Stephen A Rains. 2014. The implications of stigma and anonymity for self-disclosure in health blogs. Health Communication 29, 1 (2014), 23-31.

[54] Jeremie Richard, Icoquih Badillo-Amberg, and Phyllis Zelkowitz. 2017. "So Much of This Story Could Be Me": Men's Use of Support in Online Infertility Discussion Boards. American fournal of Men's Health 11, 3 (2017), 663-673.

[55] Lisa R Rubin, Jessica Chavez, Amy Alderman, and Andrea L Pusic. 2013. 'Use what God has given me': difference and disparity in breast reconstruction. Psychology \& health 28, 10 (2013), 1099-1120.

[56] Ian Ruthven. 2019. Making Meaning: A Focus for Information Interactions Research. In Proceedings of the 2019 Conference on Human Information Interaction and Retrieval. ACM, 163-171.

[57] A Scrapy. 2016. Fast and powerful scraping and web crawling framework. Scrapy. org. Np (2016).

[58] Chirag Shah and Roberto González-Ibáñez. 2011. Evaluating the synergic effect of collaboration in information seeking. In Proceedings of the 34th international ACM SIGIR conference on Research and development in Information Retrieval. ACM, 913-922.

[59] Jill Shawe, Dilisha Patel, Mark Joy, Beth Howden, Geraldine Barrett, and Judith Stephenson. 2019. Preparation for fatherhood: A survey of men's preconception health knowledge and behaviour in England. PLOS ONE 14, 3 (2019), $1-18$.

[60] Xiaolin Shi, Jun Zhu, Rui Cai, and Lei Zhang. 2009. User grouping behavior in online forums. In Proceedings of the 15th ACM SIGKDD international conference on Knowledge discovery and data mining. ACM, 777-786.

[61] Pauline Slade, Catharine O'Neill, Adrian J Simpson, and Hany Lashen. 2007. The relationship between perceived stigma, disclosure patterns, support and distress in new attendees at an infertility clinic. Human Reproduction 22,8 (2007), 2309-2317.

[62] British Psychological Society. 2013. Ethics guidelines for internet-mediated research. Leicester, UK: British Psychological Society (2013).

[63] Tammy Toscos, Sunny Consolvo, and David W McDonald. 2010. ... is it normal to be this sore?:: using an online forum to investigate barriers to physical activity. In Proceedings of the 1st ACM International Health Informatics Symposium. ACM, 346-355.

[64] Richard E Tyler and Sarah Williams. 2014. Masculinity in young men's health: Exploring health, help-seeking and health service use in an online environment. Journal of Health Psychology 19, 4 (2014), 457-470.

[65] Jonathan N Warner and Keith A Frey. 2013. The well-man visit: addressing a man's health to optimize pregnancy outcomes. The fournal of the American Board of Family Medicine 26, 2 (2013), 196-202.

[66] Jennifer L Welbourne, Anita L Blanchard, and Marla D Boughton. 2009. Supportive communication, sense of virtual community and health outcomes in online infertility groups. In Proceedings of the fourth international conference on Communities and technologies. ACM, 31-40.

[67] Thomas Zhang, Jason H D Cho, and Chengxiang Zhai. 2014. Understanding user intents in online health forums. In Proceedings of the 5th ACM Conference on Bioinformatics, Computational Biology, and Health Informatics. ACM, 220-229.

[68] Sue Ziebland, Alison Chapple, Carol Dumelow, Julie Evans, Suman Prinjha, and Linda Rozmovits. 2004. How the internet affects patients' experience of cancer: a qualitative study. BMF 328, 7439 (2004), 564. 